\title{
Communication
}

\section{Correcting surface coil excitation inhomogeneities in single-shot SPEN MRI}

\author{
Rita Schmidt ${ }^{\mathrm{a}, 1}$, Mor Mishkovsky ${ }^{\mathrm{b}, 1, *}$, Jean-Noel Hyacinthe ${ }^{\mathrm{c}}$, Nicolas Kunz ${ }^{\mathrm{d}}$, Rolf Gruetter ${ }^{\mathrm{b}, \mathrm{d}}$, \\ Arnaud Comment ${ }^{\mathrm{e}}$, Lucio Frydman ${ }^{\mathrm{a}, *}$ \\ a Department of Chemical Physics, Weizmann Institute of Science, Rehovot, Israel \\ ${ }^{\mathrm{b}}$ Laboratory of Functional and Metabolic Imaging, Ecole Polytechnique Fédérale de Lausanne (EPFL), Lausanne, Switzerland \\ ${ }^{\mathrm{c}}$ School of Health, University of Applied Sciences and Arts of Western Switzerland, Geneva, Switzerland \\ ${ }^{\mathrm{d}}$ Center of Biomedical Imaging (CIBM), Ecole Polytechnique Fédérale de Lausanne (EPFL), Lausanne, Switzerland \\ ${ }^{\mathrm{e}}$ Institute of the Physics of Biological Systems, Ecole Polytechnique Fédérale de Lausanne (EPFL), Lausanne, Switzerland
}

\section{A R T I C L E I N F O}

Article history:

Received 30 April 2015

Revised 25 August 2015

Available online 4 September 2015

\section{Keywords:}

Ultrafast MRI

Spatiotemporal encoding

$B_{1}$ corrections

Swept pulses

Surface coil MRI

\begin{abstract}
A B S T R A C T
Given their high sensitivity and ability to limit the field of view (FOV), surface coils are often used in magnetic resonance spectroscopy (MRS) and imaging (MRI). A major downside of surface coils is their inherent radiofrequency (RF) $B_{1}$ heterogeneity across the FOV, decreasing with increasing distance from the coil and giving rise to image distortions due to non-uniform spatial responses. A robust way to compensate for $B_{1}$ inhomogeneities is to employ adiabatic inversion pulses, yet these are not well adapted to all imaging sequences - including to single-shot approaches like echo planar imaging (EPI). Hybrid spatiotemporal encoding (SPEN) sequences relying on frequency-swept pulses provide another ultrafast MRI alternative, that could help solve this problem thanks to their built-in heterogeneous spatial manipulations. This study explores how this intrinsic SPEN-based spatial discrimination, could be used to compensate for the $B_{1}$ inhomogeneities inherent to surface coils. Experiments carried out in both phantoms and in vivo rat brains demonstrate that, by suitably modulating the amplitude of a SPEN chirp pulse that progressively excites the spins in a direction normal to the coil, it is possible to compensate for the RF transmit inhomogeneities and thus improve sensitivity and image fidelity.
\end{abstract}

(c) 2015 Elsevier Inc. All rights reserved.

\section{Introduction}

Contemporary magnetic resonance imaging (MRI) offers a variety of contrast sources that extend well beyond the classical $T_{1}$ and $\mathrm{T}_{2}$ image weightings. Physiological parameters such as $\mathrm{pH}$ and temperature can be detected and mapped using chemical exchange saturation transfer (CEST) [1,2]; hyperpolarized MRI delivers real-time measurements of metabolic substrates and/or sensitized contrast agents $[3,4]$; tissue oxygenation can be mapped in real-time functional studies via the BOLD effect [5]; morphology and abnormalities can be revealed using diffusion weighted imaging (DWI) [6] and diffusion tensor imaging (DTI) routines

Abbreviations: EPI, echo-planar imaging; FOV, field of view; MRI, magnetic resonance imaging; RF, radio frequency; SPEN, SPatiotemporal ENcoding; TE, echo time; TR, repetition time.

* Corresponding authors.

E-mail addresses: mor.mishkovsky@epfl.ch (M. Mishkovsky), lucio.frydman@ weizmann.ac.il (L. Frydman).

${ }^{1}$ Contributed equally to this study.
[7]. Although all these MRI approaches are different in nature and in their observables, they all share an important demand in common: they require ultrafast imaging protocols to record the in vivo physiological changes that they target. Various schemes have thus been proposed to reduce the time needed to acquire such MRI images. Some, like FLASH, RARE and their variants [8,9], rely on rapid repetitions of single $k$-space line acquisitions; although highly robust, these often are not fast enough to deliver the kind of information mentioned above. Such experiments are thus often done using "ultrafast" sequences, specifically designed for acquiring the entire multidimensional $k$-space in a single scan [10]. Foremost among these counts echo-planar imaging (EPI) [11]. Despite its video-rate ability EPI requires sampling its phase-encoded dimension with a relatively low effective bandwidth, leading to strong potential image distortions in inhomogeneous magnetic fields.

In recent years, hybrid spatiotemporal encoding (SPEN) schemes have been proposed as alternatives to overcome these single-scan MRI acquisition limitations [12,13]. Originating in concepts related to the acquisition of multidimensional MRS data in a 
single-scan, SPEN's low-bandwidth dimension is not bound by the Nyquist criteria that apply to EPI, and can thus provide higher immunities to $B_{0}$ inhomogeneities. Recent studies have shown these advantages with SPEN applications that included diffusion [14], functional MRI [15], perfusion imaging [16] and chemical shift spectroscopic imaging [17] studies. SPEN pulse sequences differ from their EPI counterparts in that, instead of relying on a single excitation pulse, they include linearly-swept "chirped" radiofrequency (RF) pulses applied in the presence of a magnetic field gradient. This results in a sequential excitation of the spins along the gradient's direction, leading to a uniaxial quadratic phase profile, whose "stationary point" can be displaced throughout the sample by the application of an acquisition gradient. When coupled to a regular readout gradient oscillation this provides a way of rastering the low-bandwidth dimension of a 2D image acquisition without the constraints of a Fourier transform - directly in the image domain. This offers the opportunity to obtain high-fidelity images even in inhomogeneous $B_{0}$ fields that would compromise EPI's performance $[18,19]$, as well as to selectively 'zoom' into specific regions within tissues [20] without suffering from folding artifacts.

The aim of the present study was to explore the possibility of relying on SPEN's spatially-progressive excitation scheme, also to correct for $B_{1}$ inhomogeneities across the volume of interest. Non-uniform $B_{1}$ is an intrinsic characteristic of many MRI setups, particularly those associated with surface coils [21-23]. Although the detection profile associated to such non-uniform $B_{1}$ 's can be corrected using post-acquisition image processing [34], they do not compensate for losses in SNR. This is by contrast to adjustments in the RF excitation profile, which if performed during the application of the pulse can make up for lost signal intensities. Several methods have been developed to achieve this, including the use of complex RF pulses that incorporate the $B_{1}$ profile in addition to the required slice selection [24,25], multi-channel transmit arrays allowing RF transmit control [26,27], and adiabatic $\mathrm{RF}$ pulses $[28,29]$. The latter are relatively easy to implement and do not require specialized hardware, but when incorporated into ultrafast imaging sequences such as EPI they increase further echo times that are already long due to the diffusion measurement implementation. When coupled to potential $B_{0}$ inhomogeneities producing $T_{2}^{*}$ blurring and artifacts, this often leads to a need for using multiple, segmented acquisitions [30]. As is shown here, modulating the pulse amplitude of SPEN's "chirped" encoding pulse along the main direction of the $B_{1}$ field inhomogeneity, also allows one to compensate for the RF inhomogeneity and thus improve image fidelity and sensitivity. In addition, reliance on SPEN delivers a higher robustness to $T_{2}^{*}$ blurring and to $B_{0}$-derived artifacts, enabling diffusion implementations based on single shot scans. Initial results in this area were shown by Snyder et al. [31], which included estimations of the corrections that could be expected from 2D spatiotemporal encoding sequences. In this work, we show that even 1D $B_{1}$ corrections can endow hybrid SPEN-based scans with substantial improvements when working with a surface coil. In addition, a particularly simple way of finding a suitable $B_{1}$ correction is demonstrated in both phantom and in vivo experiments using surface coils; the performances of SPEN sequences that employ this compensation are then compared against EPI experiments.

\section{Exploiting SPEN's spatial encoding for compensating $B_{1}^{+}$ inhomogeneities}

SPEN's encoding (Fig. 1) is usually implemented using a chirped pulse combined with an excitation gradient, imparting a parabolic phase to be decoded during the acquisition. If implemented in a socalled "hybrid" single-shot 2D mode, this spatial encoding acts along the low-bandwidth direction (assumed here to lie along $y$ and to extend over a field of view FOV), while a regular $k$-space encoding acts along the readout direction (taken along $x$ ). The chirped pulse waveform executing SPEN's excitation/encoding can be expressed as

$\mathrm{RF}(t)=B_{1}(t) e^{i \varphi_{1}(t)}$

where $B_{1}(t)$ defines an envelop that here will usually be a WURST40 shape $B_{1}(t) \propto\left(1-\cos ^{40}\left(\pi t / T_{\text {enc }}\right)\right)$ [32], $T_{\text {enc }}$ is the pulse duration, and $\varphi_{1}(t)=R t^{2} / 2+O_{i} t$ defines the rotating-frame phase of a linear frequency sweep, whose rate is $R=B W / T_{\text {enc }}$ and bandwidth is $\mathrm{BW}=-2 O_{i}=\gamma G_{e n c} \mathrm{FOV}$. The action of this chirped RF takes place while under the application of a gradient $G_{e n c}$; following the encoding and an eventual spin-echo pulse, the signal is then collected over a time $T_{a c q}$ while under the action of an acquisition gradient $G_{a c q}$. For simplicity we shall also assume that the $B_{1}$ inhomogeneity caused by the surface coil is one-dimensional (an assumption that is further examined below), and that its main axis also coincides with the $y$-sweep direction. Under the usual assumption that the chirped pulse excites each of the spin packets progressively along their $y$

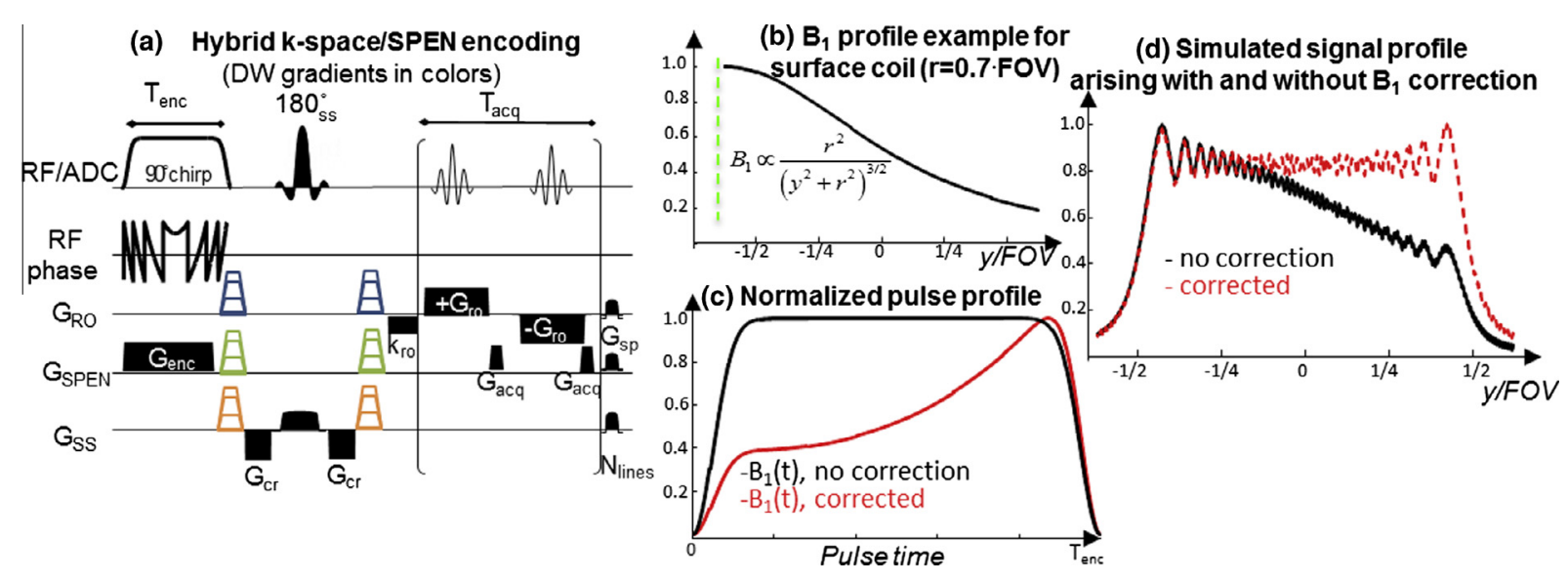

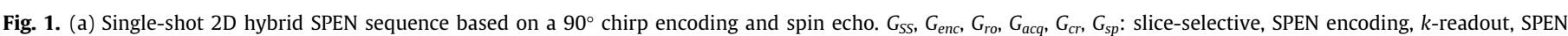

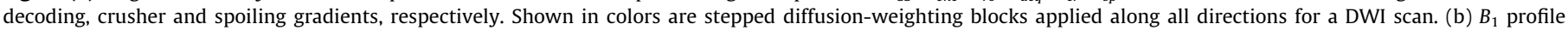

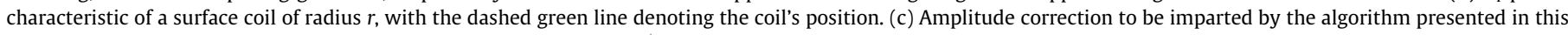

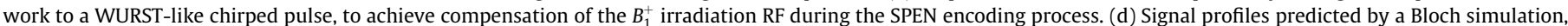

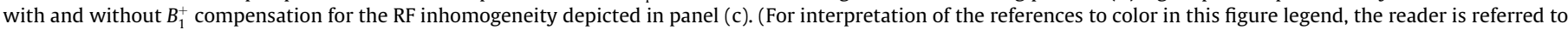
the web version of this article.) 
location, one can elaborate a signal expression to include potential $M_{+}(y)$ heterogeneities in the excited magnetization profile as well as potential $C(y)$ receiver inhomogeneities:

$S(t) \propto \int_{-\frac{\mathrm{FOV}}{2}}^{\frac{\mathrm{FOV}}{2}} C(y) M_{+}(y) \rho(y) e^{i\left(\varphi_{\text {enc }}(y)+k(t) y\right)} d y$

Here $\varphi_{\text {enc }}(y) \propto y^{2}$ is the parabolic phase imparted by the chirped encoding pulse [13], $k(t)=\gamma \int_{0}^{t} G_{a c q}\left(t^{\prime}\right) d t^{\prime}$ is the wavenumber accrued as a function of the acquisition time $t$, and $\rho(y)$ is the spin density being sought. In past SPEN analyses we had assumed uniform transmit and receive RF profiles, and thereby to $C(y) M_{+}(y)=1$. In the present instance, however, we describe the dependencies in the $R F$ transmit and receive profiles as

$C(y) M_{+}(y) \propto B_{1}^{-}(y) \sin \left(\gamma B_{1}^{+}(y) \tau\right)$

where $\tau$ is the pulse duration. Here $B_{1}^{+}(y)$ is the profile of the RF pulse imparting its effects on $M_{+}(y)$ via the sine of the excitation angle, and $B_{1}^{-}(y)$ is the receiving field sensitivity associated to the acquisition $[33,34]$.

Eq. (3) suggests that upon employing surface or other inhomogeneous coils, two attenuation/distortion sources arise: one related to the RF transmission, and the other to the reception. Since the receiving profile distortions can be compensated to some extent by post-processing [35], we focus on correcting the transmission as main goal of this study. To do so we rely again on the stationary phase approximation [13], which for a relaxation-free scenario predicts that the signal $S(t)$ at each time point will be proportional to the local spin magnetization and density, according to $S(t) \propto \sin \left(\gamma B_{1}^{+}\left(y_{0}(t)\right) \tau\right) \rho\left(y_{0}(t)\right)$ - with $y_{o}$ the coordinate fulfilling $\left[\frac{\partial \varphi_{\text {enc }}[y(t)]}{\partial y}\right]_{y=y_{o}}=0$. Therefore, if one would modify the encoding pulse WURST envelop $B_{1}(t)$ with a modulation that a priori guarantees that $\sin \left(\gamma B_{1}^{+}\left(y_{0}(t)\right) \tau\right)=1$, the resulting excitation inhomogeneities should be minimized. Fig. 1c illustrates an example of such a RF-based profile correction, based on a simplified $B_{1}$-heterogeneity model. Naturally, there is a limit to the kind of $B_{1}$-derived corrections one can apply: eventually the RF power required for the compensation would exceed the capabilities of the scanner, of the coil, or of the power that one is allowed to deposit on the subject.

To implement the correction just described, it is necessary to measure the actual $B_{1}$ profile and utilize this information in the design of the RF chirp pulse. Numerous methods have been proposed for quantitatively measuring the spatial dependence of a $B_{1}[18,19]$; however, since in our case it is only the relative variation of the profile along the $y$-axis with $B_{1}$ that is actually required, a simpler alternative consists of measuring the spatial projections $I(y)=\int_{X \text {-axis }} \rho(x, y) d x$ afforded by an array of single-shot 2D SPEN acquisitions, repeated as a function of excitation power. This can be carried out efficiently and with good robustness vis-à-vis $B_{0}$ inhomogeneity. Such set of measurements (Fig. 2I and II) can then be translated into maps of the optimum $B_{1}$ intensities that should be used - in Gauss, kHz, arbitrary db settings, or other units on which the scanner relies for its RF power handling - versus position $y$. Translating $y$-positions into $t$-excitation times as $y=-\mathrm{FOV} / 2+\mathrm{FOV}\left(t / T_{a c q}\right)$, yields then the $t$-dependent RF amplitude by which the original profile should be corrected for in order to retrieve an optimal image (Fig. 2III and IV). A point to consider in such procedure is the nature of $y_{o}$, which can in principle be start at $\pm \mathrm{FOV} / 2$. When using the scheme in Fig. 1 , it is convenient to choose the initial $y_{o}$ - which being the first point to be excited and last one to be detected will be the position most strongly affected by $T_{2}$ losses - as the position closest to the surface coil; in Fig. 2 this position would correspond to +FOV/2. SPEN-like sequences that are devoid of spatially-dependent $T_{2}$ effects like RASER [20] would be exempt from such need, or SPEN sequences relying on a swept $180^{\circ}$ pulse for the encoding would reduce it

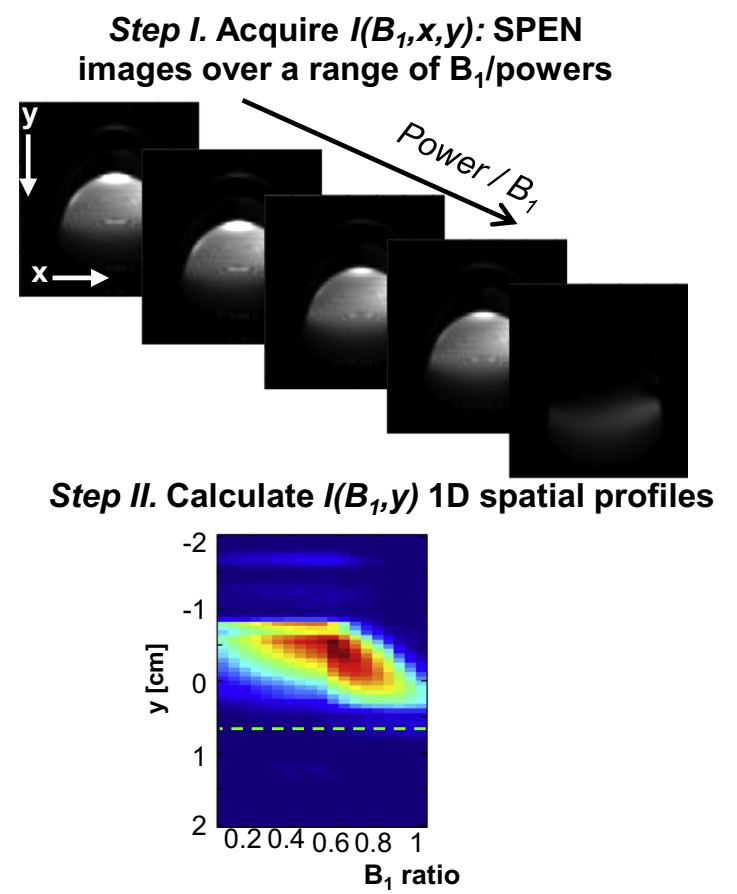

Step III. Estimate optimal $B_{1}(y)$ for achieving maximal image intensity

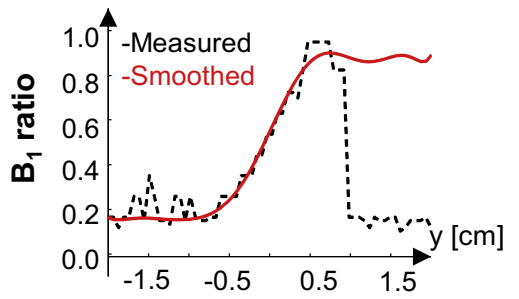

Step IV. Generate RF pulse with a shaped amplitude that includes the $B_{1}$ compensation

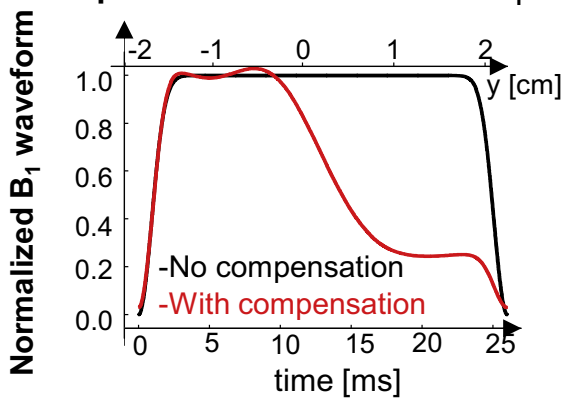

Fig. 2. Procedure used for correcting uniaxial $B_{1}$ inhomogeneities in SPEN images. Step I: a series of single-shot SPEN images are acquired over an array of RF power values. Step II: images are projected to obtain 1D spatial profiles vs normalized $B_{1}$ RF power ( $x$-axis displays $B_{1}$ ratio, defined as the applied $B_{1}$ vs maximal $B_{1}$ 'value in the set of scans, the dashed green line indicates the coil location). Step III: these $I\left(B_{1}, y\right)$ profiles are used to extract optimal $B_{1}$ powers providing maximal image intensity at each position $y$. Step IV: after suitable smoothing, the $B_{1}(y)$ dependence is translated into time over the course of the encoding according to $t=T_{a c q}(y / \mathrm{FOV}$ $+0.5)$, to obtain the final $B_{1}(t)$ amplitude modulation. Results in this experimental example involved the sequence in Fig. 1a with the following main acquisition parameters: FOV of $4 \times 4 \mathrm{~cm}^{2}$ for single slice of $2 \mathrm{~cm}$ thickness, acquisition dwell time $4 \mathrm{us}, T_{\text {enc }}=27 \mathrm{~ms}, G_{e n c}=0.6 \mathrm{G} / \mathrm{cm}, T_{a c q}=27 \mathrm{~ms}, 64 \times 64$ matrix size with inplane resolution of $0.6 \times 0.6 \mathrm{~mm}^{2}$. 
significantly [36,37]. Still, a similar procedure as the one just described would deliver the $y$-independent, maximum sensitivity profiles also for such alternative encoding schemes.

It is enlightening to assess the quality of improvements that such 1D $B_{1}$ compensation could bring about; to this end, a set of simulations was performed for an idealized surface coil setup. Since the $B_{1}$ distribution associated to a surface coil is not necessarily symmetric when tissue properties are considered [38], full 3D electromagnetic simulations were performed to assess this. Simulations of the $B_{1}$ profile in transverse and longitudinal planes were performed using the FIT $^{\circledR}$ (finite integration technique) software (CST Microwave Studio, Darmstadt, Germany), with a setup including a $15 \mathrm{~mm}$ coil radius and a phantom with brain tissue parameters. In parallel to what was later done experimentally, the examined FOV was $30 \mathrm{~mm}$ and started at a distance of $5 \mathrm{~mm}$ from the coil location. With these parameters, 2D spatial simulations of a constant and of a corrected excitation profile were then performed for transverse and longitudinal planes (Fig. 3a-d). Calculations were also performed for three kind of spin echo sequences: a $90-180^{\circ}$ scheme using sinc pulses without correction, a $90^{\circ}$ sinc excitation pulse followed by two $180^{\circ}$ adiabatic pulses, and a $90^{\circ}$ chirp pulse with $1 \mathrm{D} B_{1}$ compensation followed by an optimized $180^{\circ}$ (choosing the latter's power to obtain optimal sensitivity inside the FOV). The results expected from these sequences are summarized in Fig. 3e-g. As can be seen, even although the $B_{1}$ distribution of a surface coil positioned over tissue is not one dimensional, applying 1D corrections of the kind depicted in
Fig. 1 on these spin-echo experiments can achieve nearly ideal excitation profiles, reaching $\geqslant 95 \%$ in average intensities within the relevant FOVs for a single chirped $\alpha$-pulse. These very high performance levels, however, were not achieved in implementations like the one illustrated in Fig. 2, where the $B_{1}$ was tailored solely in the excitation and not in the full spin echo implementation. Nevertheless, simulations show that these compensated SPEN sequences offer similar sensitivity enhancements as the semiadiabatic SE-EPI sequences of the kind introduced in Ref. [30] while enjoying from the additional robustness characteristic of SPEN experiments.

\section{Experimental}

\subsection{MR measurements}

In vitro and in vivo measurements were performed on a $9.4 \mathrm{~T} / 31 \mathrm{~cm}$ actively shielded animal scanner (Magnex Scientific, Oxford, UK) equipped with a 12-cm-inner-diameter gradient (400 mT/m in $x, y, z$ directions; Magnex Scientific) and interfaced to a VNMRS ${ }^{\circledR}$ console (Varian Inc., Palo Alto CA, USA). A customdesigned quadrature ${ }^{1} \mathrm{H}$ surface coil consisting of two geometrically decoupled $16-\mathrm{mm}$ diameter single loops, was used as transmitter/receiver probe. $B_{o}$ field inhomogeneity was corrected using the FASTMAP protocol [39]. In vitro tests were carried out on tap water phantoms. In vivo measurements were performed

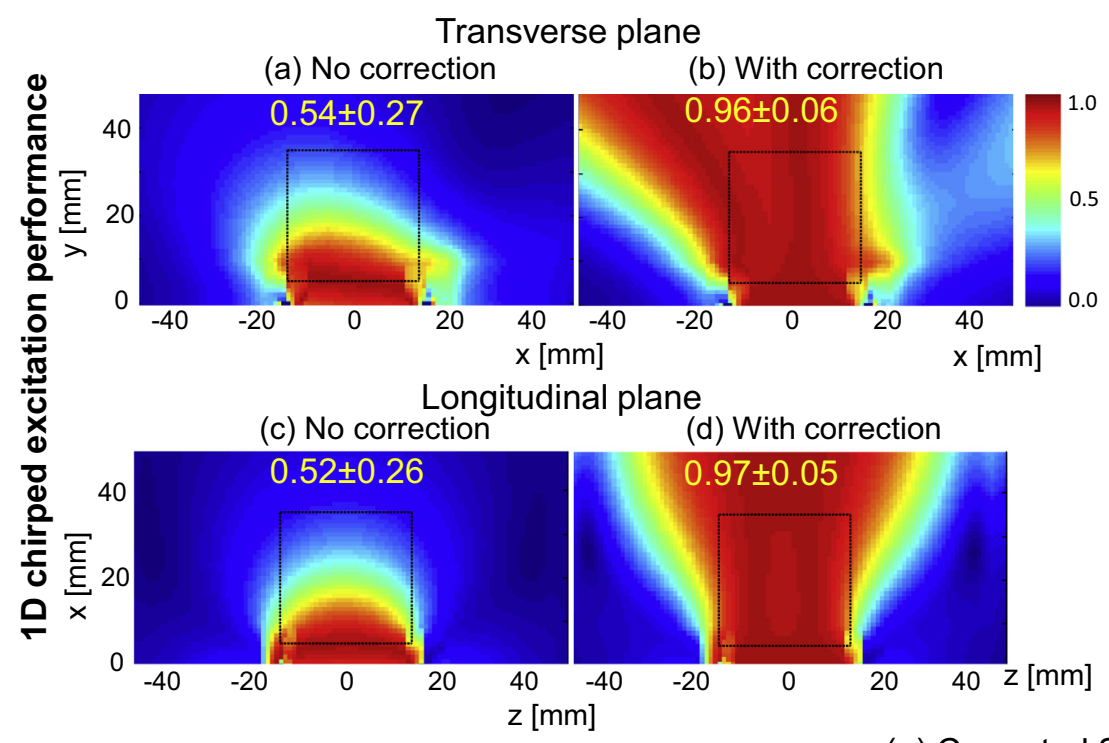

(e) $90^{\circ}-180^{\circ}$ no correction

(f) Semi-adiabatic:

(g) Corrected SPEN

$180^{\circ}$ adiabatic pulse
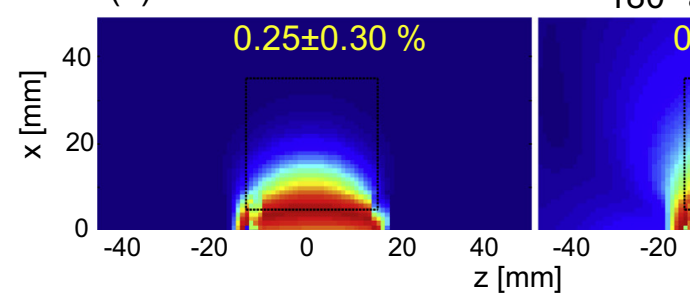
$0.52 \pm 0.26$

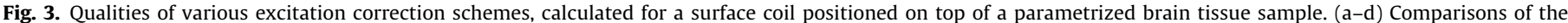

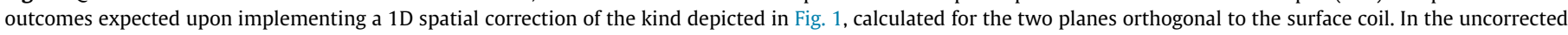

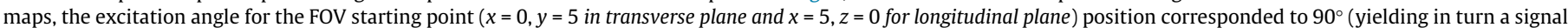

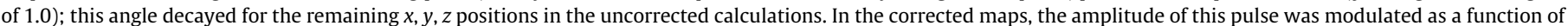

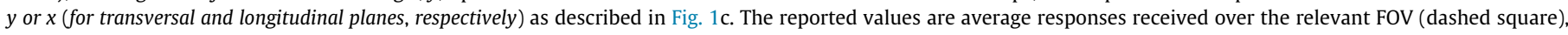

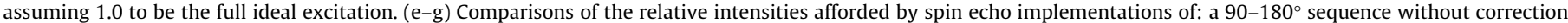

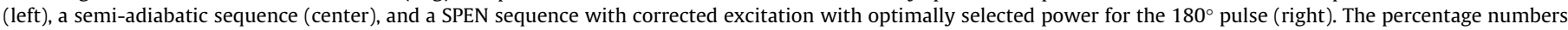
report the average signal and the signal ranges detected for each sequence inside the FOV. 
on male Sprague-Dawley rats with 350 g average weights; the animals were anesthetized using $1.5 \%$ isoflurane and their physiology was monitored throughout the scans. All experiments were approved by the local ethics committee.

\subsection{Pulse sequences and processing}

SPEN acquisitions were implemented using the sequence in Fig. 1a, with RF pulses and gradient shapes designed in Matlab ${ }^{\circledR}$ (The MathWorks Inc., Natick, MA) and uploaded onto the scanner. The SPEN image reconstruction was also performed using customwritten Matlab packages, which included a super-resolution (SR) processing of the data along the spatiotemporal dimension [40], and a conventional FT along the $k$-dimension. Pre-SR data manipulations included minor realignments of positive and negative readout echoes, as detailed in Ref. [40]. The SPEN images were compared against SE EPI measurements performed using pulse sequences provided with the Varian scanner. For the single-shot EPI tests these used a $90^{\circ}$ sinc pulse for slice selection and a slice-selective $180^{\circ}$ pulse for refocusing. Interleaved four-shot SE-EPI experiments were also done [30], using pairs of adiabatic hyperbolic secant pulses for the echoing. For the DWI measurement comparisons, pulsed field gradients were placed symmetrically around the $180^{\circ}$ refocusing inversion pulses in both SE-EPI and SPEN acquisitions. The diffusion weighing in the SPEN acquisitions was estimated taking into consideration the $b$-values dependence along the $y$-direction, as described in Ref. [14].

To evaluate the $B_{1}$ profiles and implement the corrections described in Fig. 2, a series of constant-RF-amplitude acquisitions based on WURST-40 pulse shapes was performed, while varying the maximum pulse power over 19 equally-stepped $B_{1} d b$-values. These single-scan experiments placed the spatiotemporally encoded dimension along the main inhomogeneity axis of the $B_{1}$ field. The final 1D power profile to be applied in $B_{1}$-compensated experiments was obtained by extracting for each location the $B_{1}$ that delivered maximal signal intensity, making a composite of these maximum-intensity $B_{1}(y(t))$ values, and then smoothing the ensuing waveform profile (Fig. 2-IV). Bloch-equation simulations (Fig. 1d) confirmed the accuracy of this procedure. This correction method was adopted for both in vitro and in vivo measurements.

\section{Results}

\subsection{Phantom experiments}

Fig. 4 compares spin echo SPEN images acquired on a water phantom sample, with and without the $B_{1}$-compensation procedure just described. Panel (a) illustrates how, due to the coil's inhomogeneity, increasing the maximum amplitude of the chirped pulse used to impart the SPEN, enables one to highlight progressively deeper regions along the $y$-axis, as they depart from the position of the surface coil. Average $I\left(y, B_{1}\right)$ plots (Fig. $4 \mathrm{~b}$ ) yield the RF settings that should be used for optimizing the amplitudes as a function of $y / t$; the improvements brought about by the correction in terms of spatial homogeneity and signal intensity are evidenced by the 2D image shown in Fig. 4c. As also shown in this panel, changing the maximum RF power will then scale the overall signal intensity, but will no longer introduce appreciable distortions as a function of depth. This is further illustrated in
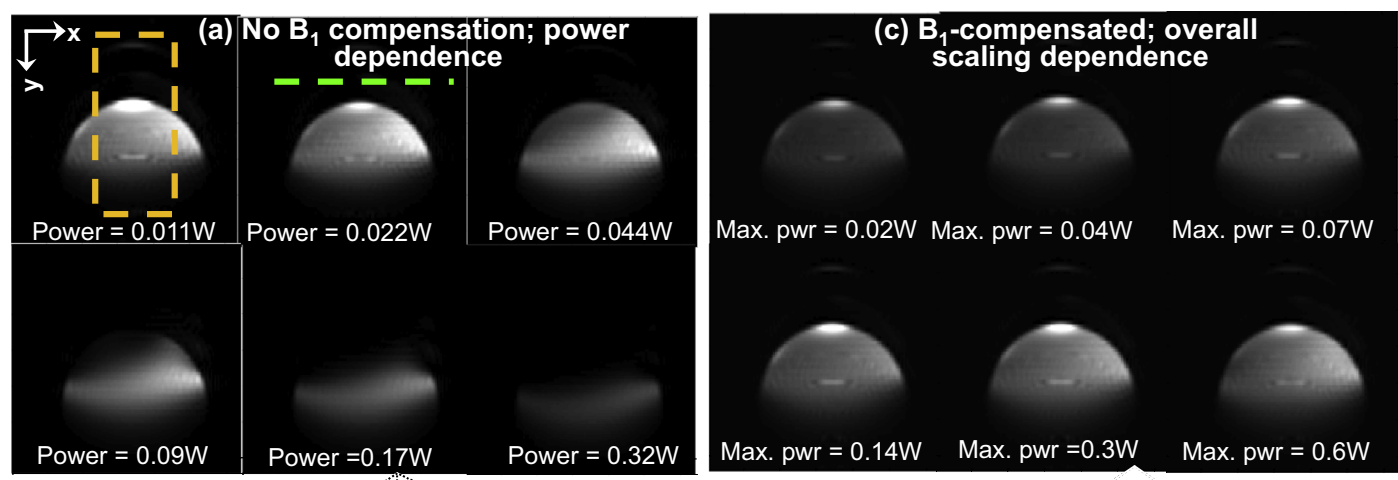

(e) Profiles compared (rf coil indicated)

(b) Average profile (boxed region); no compensation

(d) Average profile (boxed

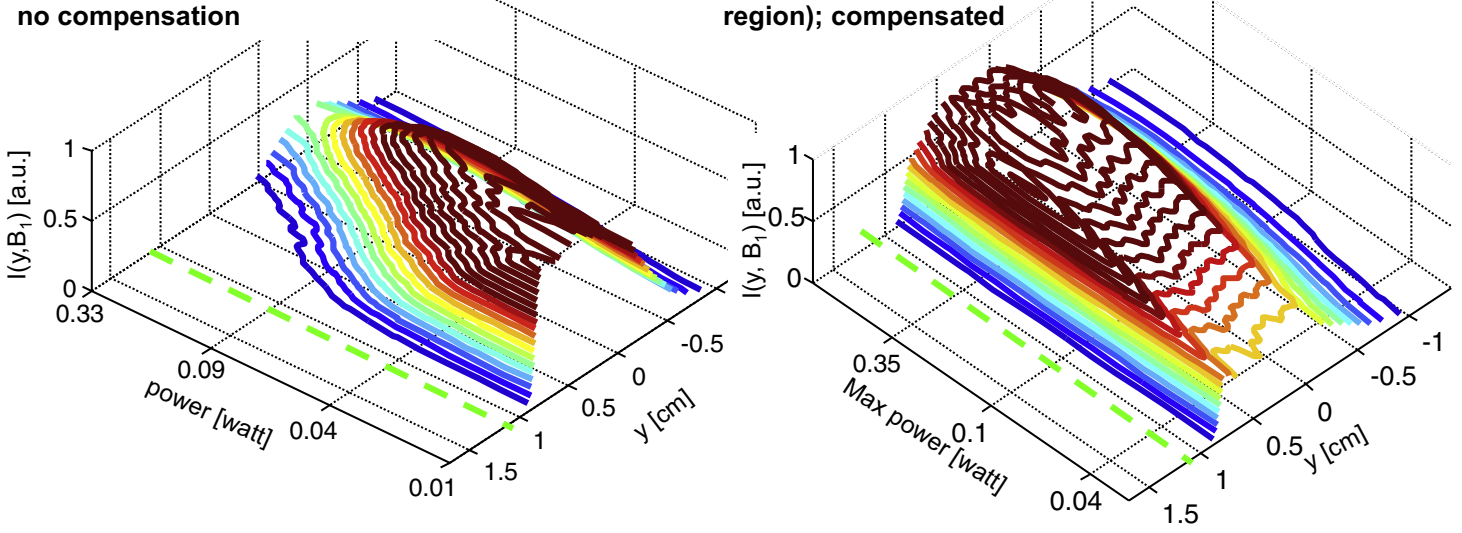

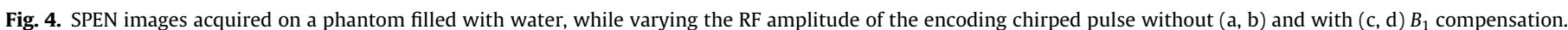

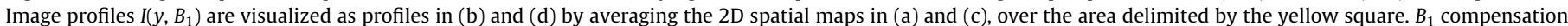

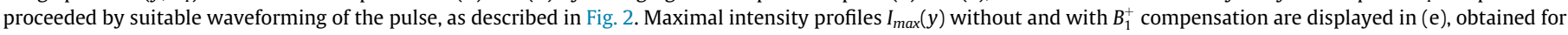

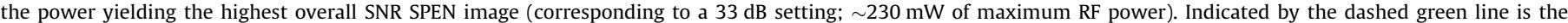

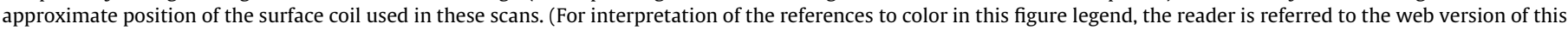
article.) 
Fig. 4d, which depicts the intensity profiles $I$ that are then observed versus power and y direction. Finally, Fig. 4e highlights the improved spatial coverage that can be obtained by this $B_{1}$ correction mode.

\subsection{In vivo measurements}

The performance of this $B_{1}$-correction approach was also evaluated in single-scan in vivo acquisitions. SE-EPI images were acquired and compared to SPEN acquisitions collected with and without the $B_{1}$ compensation algorithm, using two different approaches. One included a single-shot, regular SE-EPI sequence; the other utilized a SE-EPI incorporating two $180^{\circ}$ adiabatic refocusing pulses for enhancing the robustness vis-à-vis $B_{1}$ inhomogeneities. These measured results, shown in Fig. 5, demonstrate once again a clear improvement in FOV coverage along the main axis of the surface coil (Fig. 5d and g); this improvement results in both a higher signal sensitivity, as well as the higher fidelity that SPEN images usually display vis-à-vis EPI counterparts. As for the different contrasts evidenced by the SPEN and EPI images, these are most likely owing to the different $T_{2}$ weightings of the two experiments [13].

One of the promising applications of SPEN is DWI - particularly at high fields or in heterogeneous tissues liable to susceptibility distortions $[14,41,42]$. To explore the potential improvements that the $B_{1}$ corrected SPEN scheme hereby introduced could bring to DWI, single scan experiments were acquired on a rat brain with the same surface coil setup as in Fig. 5, for different $b$-values. These images were compared to comparable data arising from singlescan diffusion-weighted SE-EPI experiments. As once again evidenced in Fig. 6, larger FOVs along the $y$-direction endowed with better sensitivity, could be achieved thanks to the enhanced coverage of the corrected SPEN procedure. Calculated apparent diffusion coefficient (ADC) maps obtained from both schemes were nevertheless similar in regions where quality signals were available. These results evidence another potential advantage of SPEN for this kind of DWI investigations.

\section{Discussion and conclusions}

The present study explored a simple approach to correct for uniaxial RF inhomogeneity distortions, of the kind that normally will arise upon operating with a single transmit/receive surface coil. It was shown that high-SNR and high-fidelity single-shot MR images can then be acquired, by exploiting the coaxiality between the distortions introduced by an uneven $B_{1}$ excitation, and the spatiotemporal encoding process executed by a frequency-swept chirp pulse. By performing a rapid series of SPEN calibration measurements based on scans as a function of $B_{1}$ value, power levels capable of offsetting the dropping $B_{1}$ s associated to the use of a surface coil could be found. When considering the kind of distortions associated to the use of surface coils, which are liable to be load-dependent and hence in need of precalibrations for different samples, the present method is particularly convenient. Incorporating such predetermined $B_{1}$ s into a revised chirped excitation profile extended the achievable FOV coverage and increased the overall signal, in a relatively simple fashion. These improvements were evidenced by both in vitro and in vivo tests, including a 2D diffusion-weighted imaging study of a rat brain that demanded a single $27 \mathrm{~ms}$ chirp pulse with only $130 \mathrm{~mW}$ of mean RF power - well suited to a majority of surface coil assemblies. The ensuing spatial coverage and sensitivity of the SPEN-derived ADC maps, compared then favorably with those arising from the EPI images. This not only resulted from the new $B_{1}$ correcting procedure, but also from SPEN's already-reported robustness to $B_{0}$ inhomogeneities.

The present study focused on a particularly simple geometrical distortion of the $B_{1}$ profile, which could then be compensated by a particularly simple and rapid calibration/correction procedure. More complex procedures and extensions to alternative geometries, could naturally be conceived. In terms of surface coil distortions, which are liable to be load-dependent and hence in need of precalibrations for different samples, the present method is particularly convenient. Alternatives could include making an actual map of
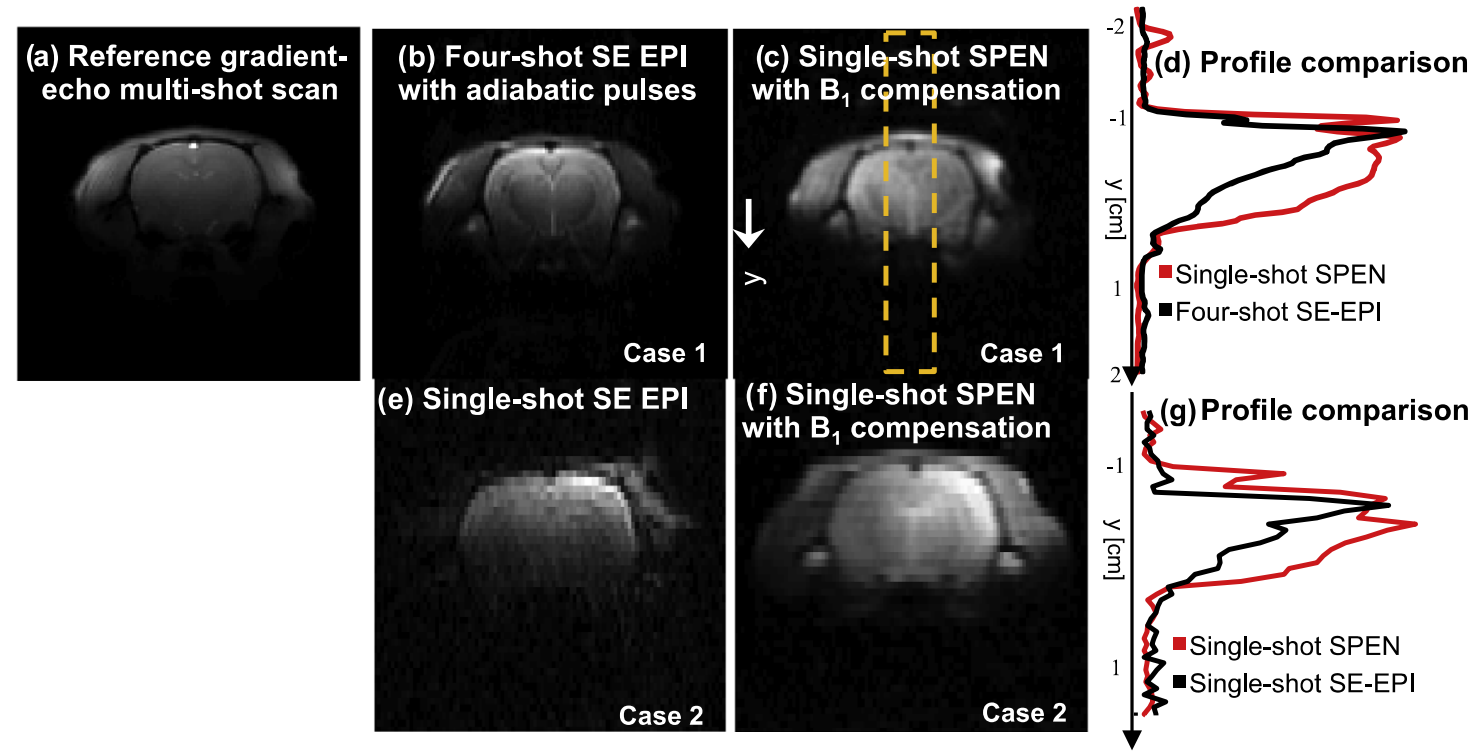

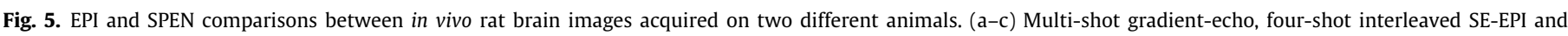

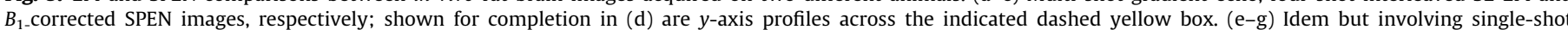

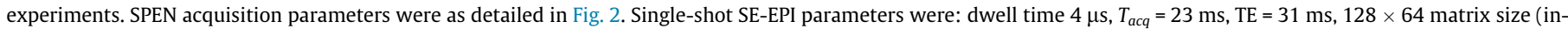

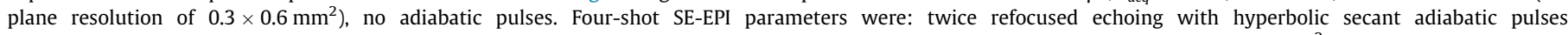

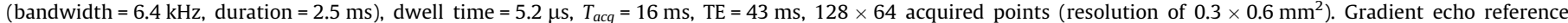

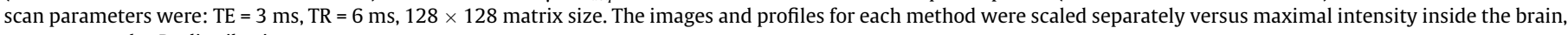
to represent the $B_{1}$ distribution. 
(a) SE-EPI DWI
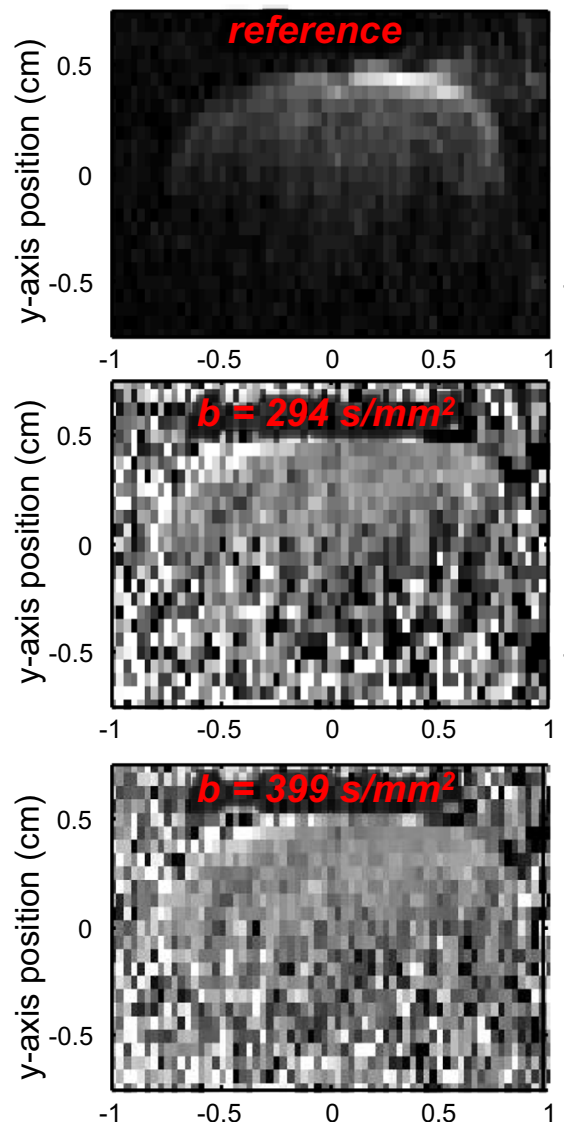

$-1$

\section{(b) $B_{1}$-corrected SPEN DWI}
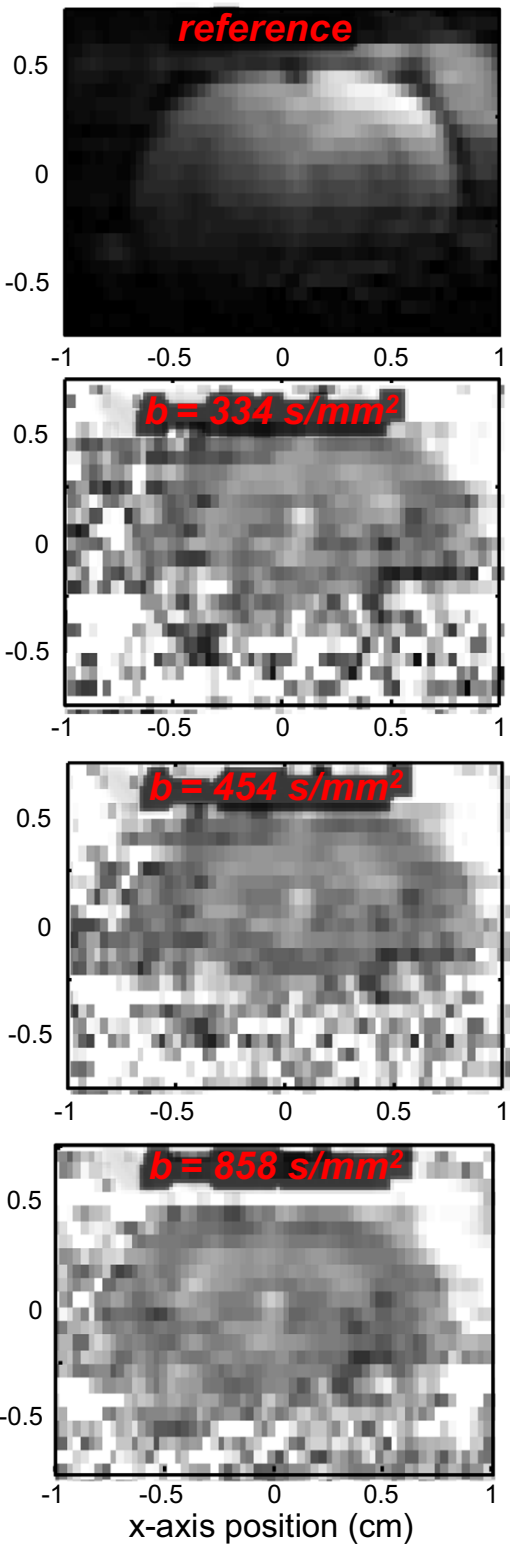

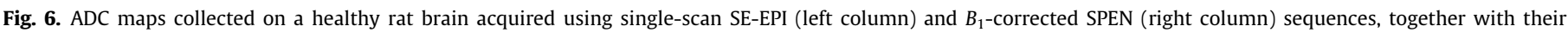

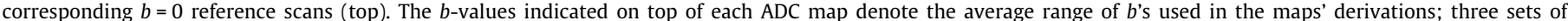

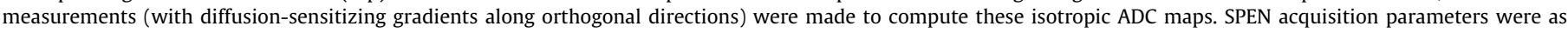

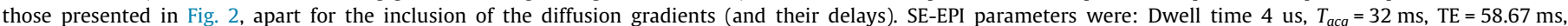

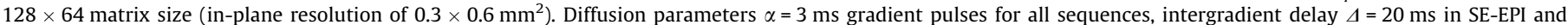
$\Delta=16.9 \mathrm{~ms}$ in SPEN.

the nutation frequencies in a two-dimensional plane, and derive the corrections to be performed from there. Indeed, although in the present work the $B_{1}$ correction was performed along a single axis, the method can be extended to obtain planar compensation by implementing suitably adapted 2D spatiotemporal RF pulses [43,31]. Such improvements might be unjustified in the simple surface-coil scenario hereby treated, yet Garwood et al. have shown their worthiness in scenarios including high-field cases where the object being targeted is sited in inhomogeneous $B_{o}$ and $B_{1}$ fields, which could then be simultaneously compensated by amplitude and phase manipulations of the chirped pulse [31]. Yet another possibility could rest in departing from the use of a constant-rate chirp pulse, and tailor the rate of the sweep $R$ to the actual strength of the $B_{1}$ value: as the spins' nutation angle is proportional to $B_{1} \sqrt{ } R$, regions of weakening
$B_{1} s$ could be excited equally well by slower sweeps. Such procedures have indeed been demonstrated in SPEN acquisitions [12]; their potential drawbacks include longer excitation times, and uneven spatial resolutions as a function of acquisition time. Alternatively VERSE-like approaches could be adopted [44] whereby the $G_{\text {enc }}$ gradient is reduced as a constant-rate chirp progresses, with similar advantages and drawbacks. Clearly, several interesting avenues arise in this area.

\section{Acknowledgments}

We are grateful to A. Seginer for helpful discussions, to A. Capozzi and Y. Pilloud for their help in designing the hardware used for the MR measurements, and to Prof. Andrew Webb for 
the facilities used to run the electromagnetic simulations. This work was supported by ERC PoC Grant \# 633888, the Israel Science Foundation ISF Grant 795/13, the Kimmel Institute of Magnetic Resonance, the generosity of the Perlman Family foundation, the Swiss National Science Foundation (grant PP00P2_157547), the Centre d'Imagerie BioMédicale (CIBM) of the UNIL, UNIGE, HUG, CHUV, EPFL, the Leenards and Jeantet Foundations, and EU COST action TD1103.

\section{References}

[1] E. Vinogradov, A.D. Sherry, R.E. Lenkinski, CEST: from basic principles to applications, challenges and opportunities, J. Magn. Reson. 229 (2013) 155172.

[2] P.C. Van Zijl, N.N. Yadav, Chemical exchange saturation transfer (CEST): what is in a name and what isn't?, Magn Reson. Med. 65 (2011) 927-948.

[3] F.A. Gallagher, M.I. Kettunen, S.E. Day, De-En Hu, J.-H. Ardenkjær-Larsen, R Zandt, P.R. Jensen, M. Karlsson, K. Golman, M.H. Lerche, K.M. Brindle, Magnetic resonance imaging of $\mathrm{pH}$ in vivo using hyperpolarized 13C-labelled bicarbonate, Nature 453 (2008) 940-943.

[4] A. Comment, M.E. Merritt, Hyperpolarized magnetic resonance as a sensitive detector of metabolic function, Biochemistry 53 (47) (2014) 7333-7357.

[5] S. Ogawa, T.M. Lee, A.R. Kay, D.W. Tank, Brain magnetic resonance imaging with contrast dependent on blood oxygenation, Proc. Natl. Acad. Sci. 87 (24) (1990) 9868-9872.

[6] D. LeBihan, E. Breton, D. Lallemand, P. Grenier, E. Cabanis, M. Laval-Jeantet, MR imaging of intravoxel incoherent motions: application to diffusion and perfusion in neurologic disorders, Radiology 161 (1986) 401-407.

[7] P.J. Basser, J. Mattiello, D. LeBihan, MR diffusion tensor spectroscopy and imaging, Biophys J. 66 (1) (1994) 259-267.

[8] A. Haase, Snapshot flash MRI. Applications to T1, T2 and chemical-shift imaging, Magn. Reson. Med. 13 (1990) 77-89.

[9] J. Hennig, A. Nauerth, H. Friedburg, RARE imaging: a fast imaging method for clinical MR, Magn. Reson. Med. 3 (1986) 823-833.

[10] F. Schmidt, M.K. Stehling, R. Turner, Echo Planar Imaging: Theory, Technique and Application, Springer, Berlin, 1998.

[11] P. Mansfield, Multi-planar image formation using NMR spin echoes, J. Phys. C: Solid State Phys. 10 (1977) L55-L58.

[12] Y. Shrot, L. Frydman, Spatially-encoded NMR and the acquisition of 2D magnetic resonance images within a single scan, J. Magn. Reson. 172 (2005) $179-190$.

[13] A. Tal, L. Frydman, Single-scan multidimensional magnetic resonance, Prog. Nucl. Magn. Reson. Spectrosc. 57 (2010) 241-292.

[14] E. Solomon, N. Shemesh, L. Frydman, Diffusion weighted MRI by spatiotemporal encoding: analytical description and in vivo validations, J. Magn. Reson. 232 (2013) 76-86.

[15] U. Goerke, M. Garwood, K. Ugurbil, Functional magnetic resonance imaging using RASER, Neuroimage 54 (1) (2011) 350-360.

[16] A. Seginer, R. Schmidt, A. Leftin, E. Solomon, L. Frydman, Referenceless reconstruction of spatiotemporally encoded imaging data: principles and applications to real-time MRI, Magn. Reson. Med. 72 (2014) 1687-1695.

[17] A. Tal, L. Frydman, Spectroscopic imaging from spatially-encoded single-scan multidimensional MRI data, J. Magn. Reson. 189 (2007) 46-58.

[18] A. Tal, L. Frydman, Spatial encoding and the single-scan acquisition of high definition MR images in inhomogeneous fields, J. Magn. Reson. 181 (2006) 179-194.

[19] J. Li, L. Chen, S. Cai, C. Cai, J. Zhong, Z. Chen, Imaging with referenceless distortion correction and flexible regions of interest using single-shot biaxial spatiotemporally encoded MRI, NeuroImage 105 (2015) 93-111.

[20] R. Chamberlain, J.Y. Park, C. Corum, E. Yacoub, K. Ugurbil, C.R. Jack, M. Garwood, RASER: a new ultrafast magnetic resonance imaging method, Magn. Reson. Med. 58 (4) (2007) 794-799.
[21] L. Axel, Surface coil magnetic resonance imaging, J. Comput. Asist. Tomogr. 8 (1984) 381-384.

[22] J.T. Vaughan, M. Garwood, C.M. Collins, W. Liu, L. Delabarre, G. Adriany, P. Andersen, H. Merkle, R. Goebel, M.B. Smith, K. Ugurbil, 7T vs. 4T: RF power homogeneity, and signal-to-noise comparison in head images, Magn. Reson. Med. 46 (2001) 24-30.

[23] P.-F. Van de Moortele, C. Akgun, G. Adriany, S. Moeller, J. Ritter, C.M. Collins, M. B. Smith, J.T. Vaughan, K. Ugurbil, B1 destructive interferences and spatia phase patterns at 7T with a head transceiver array coil, Magn. Reson. Med. 54 (2005) 1503-1518.

[24] S. Saekho, F.E. Boada, D.C. Noll, V.A. Stenger, Small tip angle three-dimensional tailored radiofrequency slab-select pulse for reduced b1 inhomogeneity at $3 \mathrm{~T}$, Magn. Reson. Med. 53 (2005) 479-484.

[25] C.Y. Yip, J.A. Fessler, C.N. Douglas, Iterative RF pulse design for multidimensional, small-tip-angle selective excitation, Magn. Reson. Med. 54 (2005) 908-917.

[26] C.M. Collins, W. Liu, J. Swift, M.B. Smith, Combination of optimized transmit arrays and some receive array reconstruction methods can yield homogeneous images at very high frequencies, Magn. Reson. Med. 54 (2005) 1327-1332.

[27] U. Katscher, P. Bornert, C. Leussler, J.S. van den Brink, Transmit SENSE, Magn. Reson. Med. 49 (2003) 144-150.

[28] R.A. de Graaf, Y. Luo, M. Terpstra, H. Merkle, M. Garwood, A new localization method using an adiabatic pulse, BIR-4, J. Magn. Reson. 106 (3) (1995) 245252.

[29] M. Garwood, K. Ugurbil, A.R. Rath, M.R. Bendall, B.D. Ross, S.L. Mitchell, H. Merkle, Magnetic resonance imaging with adiabatic pulses using a single surface coil for RF transmission and signal detection, Magn. Reson. Med. 9 (1989) 25-34.

[30] Y. Looij, N. Kunz, P. Huppi, R. Gruetter, S. Sizonenko, Diffusion tensor echo planar imaging using surface coil transceiver with a semiadiabatic RF pulse sequence at 14.1T, Magn. Reson. Med. 65 (2011) 732-737.

[31] A.L.S. Snyder, C.A. Corum, S. Moeller, N.J. Powell, M. Garwood, MRI by steering resonance through space, Magn. Reson. Med. 72 (2013) 49-58.

[32] E. Kupce, R. Freeman, An adaptable NMR broadband decoupling scheme Chem. Phys. Lett. 250 (1996) 523-527.

[33] D.I. Hoult, The principle of reciprocity in signal strength calculations - a mathematical guide, Concepts Magn. Reson. 4 (2000) 173-187.

[34] C.M. Collins, M.B. Smith, Signal-to-noise ratio and absorbed power as functions of main magnetic field strength, and definition of "90' RF pulse for the head in the birdcage coil, Magn. Reson. Med. 45 (2001) 684-691.

[35] B. Belaroussi, J. Milles, S. Carme, Y.M. Zhu, H. Benoit-Cattin, Intensity nonuniformity correction in MRI: existing methods and their validation, Med. Image Anal. 10 (2006) 234-246.

[36] N. Ben-Eliezer, L. Frydman, Spatiotemporal encoding as a robust basis for fast three-dimensional in vivo MRI, NMR Biomed. 24 (2011) 1191-1201.

[37] R. Schmidt, L. Frydman, New spatiotemporal approaches for fully-refocused, multi-slice ultrafast 3D MRI, Magn. Reson. Med. 71 (2) (2013) 711-722.

[38] P. Wardenier, Local intensity shift artifact (LISA) (abstr), Book of abstracts: Society of Magnetic Resonance in Medicine 1989, Society of Magnetic Resonance in Medicine, Berkeley, California, 1989, p. 1175.

[39] R. Gruetter, I. Tkac, Field mapping without reference scan using asymmetric echo-planar techniques, Magn. Reson. Med. 43 (2000) 319-323.

[40] N. Ben-Eliezer, M. Irani, L. Frydman, Super-resolved spatially-encoded singlescan 2D MRI, Magn. Reson. Med. 63 (6) (2010) 1594-1600.

[41] A. Leftin, J.T. Rosenberg, E. Solomon, F. Bejarano, S.C. Grant, L. Frydman Ultrafast in vivo diffusion imaging of stroke at $21.1 \mathrm{~T}$ by spatiotemporal encoding, Magn. Reson. Med. 73 (2015) 1483-1489.

[42] E. Solomon, N. Nissan, E. Furman-Haran, A. Seginer, M. Shapiro-Feinberg, H Degani, L. Frydman, Overcoming limitations in diffusion-weighted MRI of breast by spatio-temporal encoding, Magn. Reson. Med. (2015), http://dx.doi. org/10.1002/mrm.25344.

[43] J.-N. Dumez, L. Frydman, Multidimensional excitation pulses based on spatiotemporal encoding concepts, J. Magn. Reson. 226 (2013) 22-34.

[44] S. Conolly, D. Nishimura, A. Macovski, G. Glover, Variable-rate selective excitation, J. Magn. Reson. 78 (1988) 440-458. 